\title{
DCIS (Ductal carcinoma in situ) - should we call it a cancer?
}

\section{Opinion}

Ductal carcinoma in situ (DCIS) is the fourth most common cancer diagnosis in females. ${ }^{1}$ The earliest stages of cancers are known as "carcinoma in situ". Carcinoma refers to "cancer" and in situ implies "in the original place." DCIS is a non-invasive pre-cancer, where the abnormal cells are present in the lining of the breast milk duct. It is a stage 0 breast cancer. DCIS isn't life-threatening itself unless it develops into an invasive breast cancer later. Yet in conventional medicine, DCIS is often treated immediately with surgery (mastectomy or lumpectomy), radiation therapy, hormonal therapy, chemotherapy, or a combination of these treatments for abnormalities that might otherwise not even have caused an invasive breast cancer.

Ideally, breast cancer screening is supposed to find potentially lethal breast cancers, which would allow best possible treatments and translate into fewer breast cancer deaths. This certainly led to phenomenal screening programs. Often screening mammography overdiagnoses breast cancer. With overdiagnosis is overtreatment. Many of cancer therapies are deleterious to healthy tissue. If DCIS is most likely to be linked with overdiagnosis whereas an invasive cancer is not diagnosed correctly, is DCIS really a cancer? Of course, the primary goal is not less diagnosis, but the key is better treatment decision tools. Yes, while it could be true that DCIS could progress into an invasive lesion, treating DCIS as an invasive cancer could be hazardous to patients. We have to understand that although detecting a small cancer can save lives, screening may detect abnormalities as DCIS that may never be life-threatening. ${ }^{2}$

When women were surveyed and asked to make a decision about the treatment of DCIS in hypothetical scenarios, more women chose treatment as the chances of invasion increased. A $1 \%$ chance of DCIS becoming invasive translated into $42 \%$ women choosing treatment whereas a $33 \%$ chance of invasion translated into $78 \%$ women choosing treatment. ${ }^{3}$

Overdiagnosis often leads to screen-detected malignancy that would not have progressed to clinical or symptomatic presentation in a patient's lifetime and would not have harmed the patient in the absence of screening. ${ }^{4}$ The dilemma is that mammography screening programs cannot differentiate between hazardous and undamaging breast cancer. The more mammograms you do, the more cancer you might find. The overall death rate from breast cancer does not seem to decline much. Moreover, very few well-done studies exist observing the progression of DCIS. Do DCIS go away spontaneously and how often? Is conventional medicine really treating DCIS the right way?

Oncologists assume that DCIS lesions are precursors of cancer and that early removal and treatment would decrease cancer incidence and mortality. Many long-term epidemiology studies show that the removal of even thousands of DCIS lesions annually does not lower the incidence of invasive breast cancers. An observational study of over 100,000 women with a diagnosis of DCIS, indicated that the risk of dying from breast cancer is low. Moreover, radiation therapy followed by lumpectomy treatment does not lower breast cancer mortality. ${ }^{5}$

\author{
Volume II Issue 2 - 2018 \\ Virender Sodhi \\ Ayurvedic and Naturopathic Medical Clinic, USA
}

Correspondence: Virender Sodhi,Ayurvedic and Naturopathic Medical Clinic, 2115 I I 2th Ave. NE \#4, Bellevue,WA 98004, USA, Tel 42545380 22, Fax 4254531408 ,

Email drvsodhi@ayurvedicscience.com

Received: April 05, 2018 | Published: April 18, 2018

In fact, researchers found that in women with low-grade DCIS, the ten-year breast cancer specific survival rate was $98.8 \%$ for no excision in comparison to $98.6 \%$ with surgical excision. ${ }^{6}$ This implies that among women who chose to not undergo surgery, $1.2 \%$ died of breast cancer within a decade. In the same decade, those that did undergo surgery, $1.4 \%$ died. Surgery does not seem to promote longevity in patients with DCIS.

An interesting but perplexing coincidence is that DCIS and invasive breast cancers share common risk factors, including family history of breast cancer, increased breast mass, obesity, absence of pregnancy or late age at first pregnancy. Furthermore, those with deleterious mutations of BRCA1 and 2 have a greater risk of DCIS.

However, with the overdiagnosis and overtreatment of DCIS, there still hasn't been a decrease in the diagnosis of invasive breast cancers regardless of the screening. This observation indicates that screening measures are not catching many invasive breast cancers or that the presence of DCIS distracts from high-risk lesions. Not only are unnecessary treatments being given to low-risk lesions, high-risk lesions are not being addressed, allowing for those tumors to progress.

There does not seem to be a clear link between DCIS and a progression to invasive breast tissues. Some thoughts include paracrine regulation on the microenvironment around DCIS lesions, creating a pro-oncology environment. Pathology of DCIS does not seem to give any clue as to how DCIS lesions could morph into invasive lesions. Much of this dilemma occurs because there is no gold standard marker that differentiates DCIS from any other invasive cancer.

A solution may be to place women on frequent DCIS lesion surveillance without using invasive technology (like mammography) which leads to more cancers itself. Lesions that would progress could be quickly treated while DCIS tissues that may completely resolve themselves can be left to allow the body naturally to take care of abnormalities. $^{7}$

I often say that we all have cancer cells at one point in our lives, which does not mean cancer has to kill us. Our immune system is our body's natural intelligence (Figure 1). It is very vigilant and is always on the surveillance to detect, kill, or modify abnormal cells. As a Naturopathic and Ayurvedic physician, I have been making this 
statement for more than three decades, but I was ridiculed. All of a sudden, in the last decade, we have invented immunotherapies for cancer treatments. Again, we are looking for one magic bullet and which should eradicate it all. This model is also losing its steam, as after some time, the specialized immunotherapy is losing its efficacy due to the fact that cancer cells are adapting and mutating to make immunotherapy ineffective. Furthermore, most immunotherapies concentrate on one or two immune cells, blind to any relationships between immune cells and surrounding tissue cells. As regards to DCIS, I question should we call it cancer? The word cancer has its own negative connotation, leads to undue worrying, and it may become a cause of cancer. The basic tenets of good health are: intention to stay healthy, nutrition, lifestyle changes, yoga, breathing, enjoying mother nature, good sleep, and spending quality time with your loved ones.

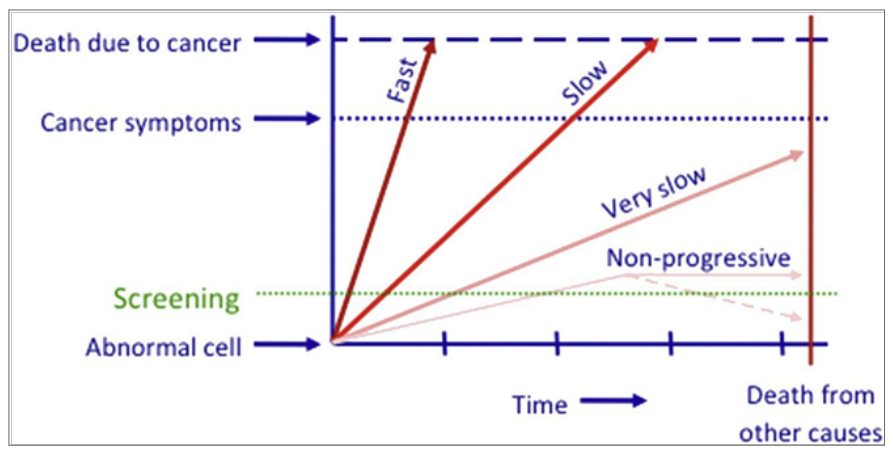

Figure I Modified figure of the heterogeneity of cancer progression. ${ }^{6}$

\section{Acknowledgement}

The author would like to thank Jessica Bedi and Richa Kumar Sood for their assistance in writing and editing.

\section{Conflict of interest}

Author declares there is no conflict of interest.

\section{References}

1. Rochman S. The DCIS Dilemma. Cancer Today. 2015.

2. Morris E, Feig S, Drexler M, et al. Implications of Overdiagnosis: Impact on Screening Mammography Practices. Popul Health Manag. 2015;18(Suppl 1):S3-S11.

3. Schwartz LM, Woloshin S, Sox HC, et al. US women's attitudes to falsepositive mammography results and detection of ductal carcinoma in situ: cross-sectional survey. BMJ. 2000; 320(7250):307-312.

4. Houssami N. Overdiagnosis of breast cancer in population screening: does it make breast screening worthless? Cancer Biol Med. 2017;14(1):1-8.

5. Esserman L, Yau C. Rethinking the Standard for Ductal Carcinoma In Situ Treatment. JAMA Oncol. 2015;1(7):881-883.

6. Groen EJ, Elshof LE, Visser LL. Finding the balance between over- and under- treatment of ductal carcinoma in situ (DCIS). Breast. 2017;274283. 